\title{
Helical Metallohost-Guest Complexes via Site-Selective Transmetalation of Homotrinuclear Complexes
}

\author{
Shigehisa Akine, Takanori Taniguchi, and Tatsuya Nabeshima* \\ Department of Chemistry, University of Tsukuba, Tsukuba, Ibaraki 305-8571, Japan \\ Supporting Information
}



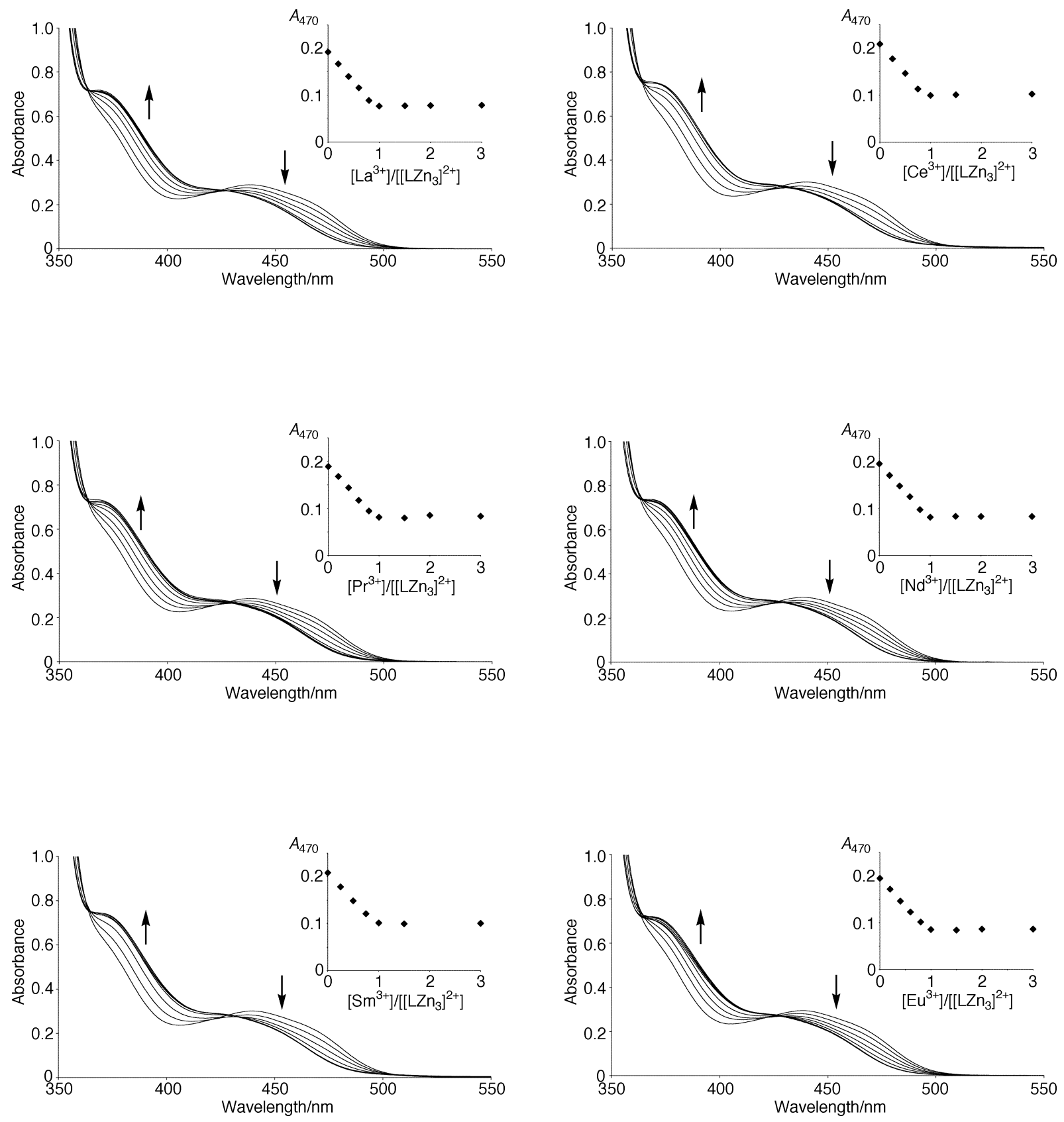

Figure S1. Spectrophotometric titration for the complexation between $\left[\mathrm{LZn}_{3}\right]^{2+}$ and $\mathrm{G}\left(\mathrm{NO}_{3}\right)_{3}(\mathrm{G}=$ $\mathrm{La}, \mathrm{Ce}, \mathrm{Pr}, \mathrm{Nd}, \mathrm{Sm}, \mathrm{Eu})$ in chloroform/methanol (1:1). Concentration of $\left[\mathrm{LZn}_{3}\right]^{2+}$ is $0.1 \mathrm{mM}$. 

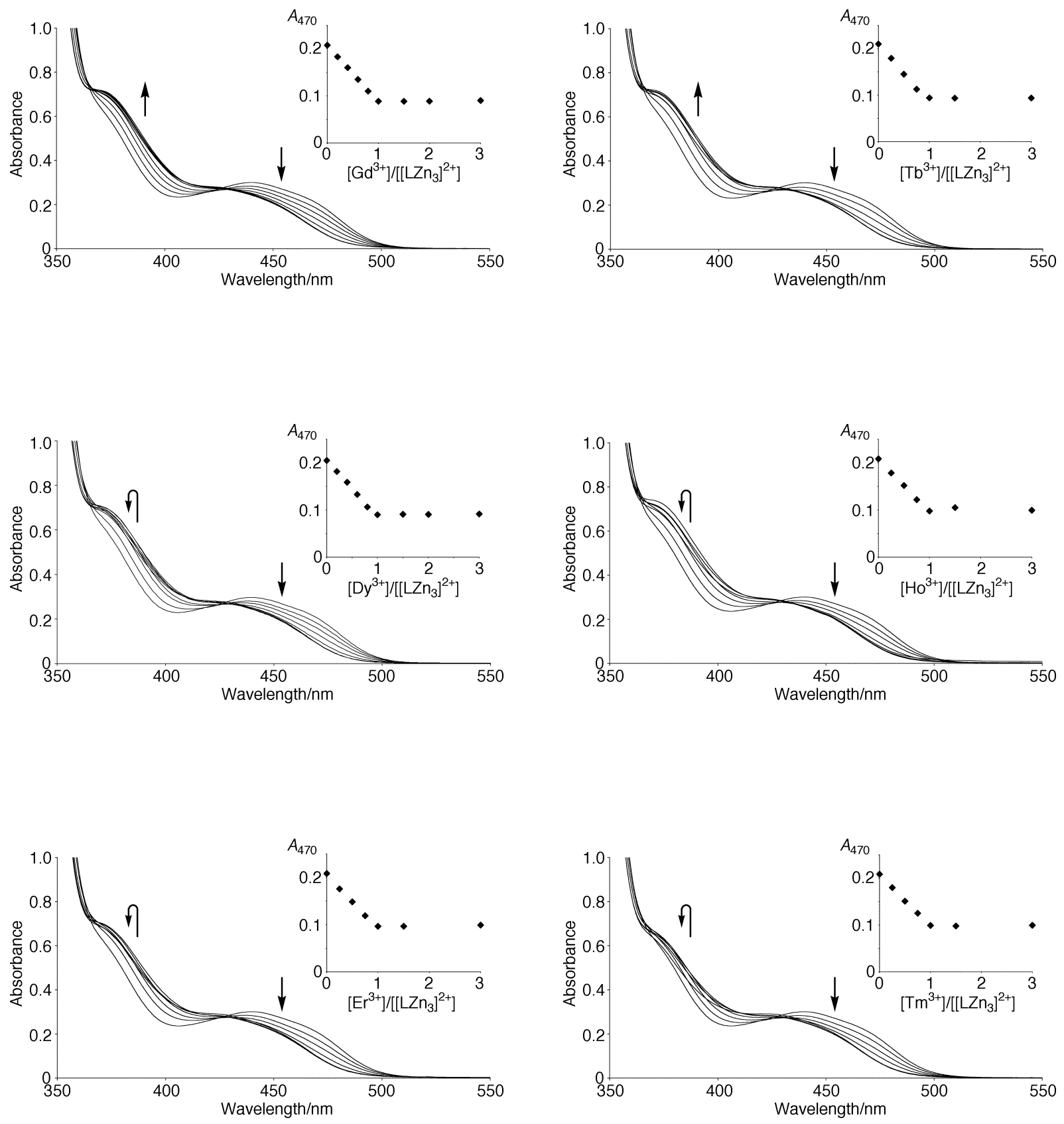

Figure S2. Spectrophotometric titration for the complexation between $\left[\mathrm{LZn}_{3}\right]^{2+}$ and $\mathrm{G}\left(\mathrm{NO}_{3}\right)_{3}(\mathrm{G}=$ $\mathrm{Gd}, \mathrm{Tb}, \mathrm{Dy}, \mathrm{Ho}, \mathrm{Er}, \mathrm{Tm})$ in chloroform/methanol (1:1). Concentration of $\left[\mathrm{LZn}_{3}\right]^{2+}$ is $0.1 \mathrm{mM}$. 

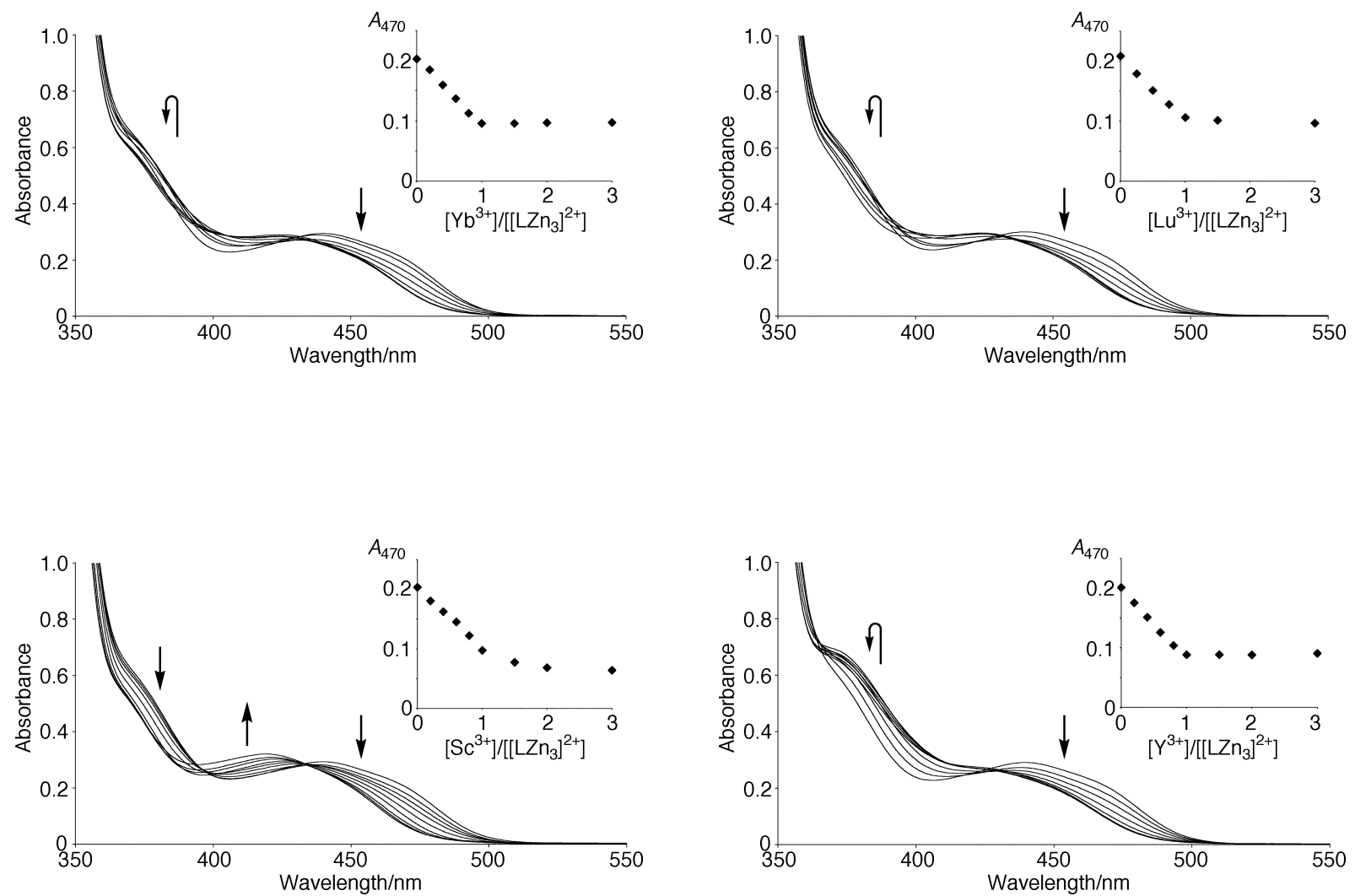

Figure S3. Spectrophotometric titration for the complexation between $\left[\mathrm{LZn}_{3}\right]^{2+}$ and $\mathrm{G}\left(\mathrm{NO}_{3}\right)_{3}(\mathrm{G}=$ $\mathrm{Yb}, \mathrm{Lu}, \mathrm{Sc}, \mathrm{Y})$ in chloroform/methanol (1:1). Concentration of $\left[\mathrm{LZn}_{3}\right]^{2+}$ is $0.1 \mathrm{mM}$. 

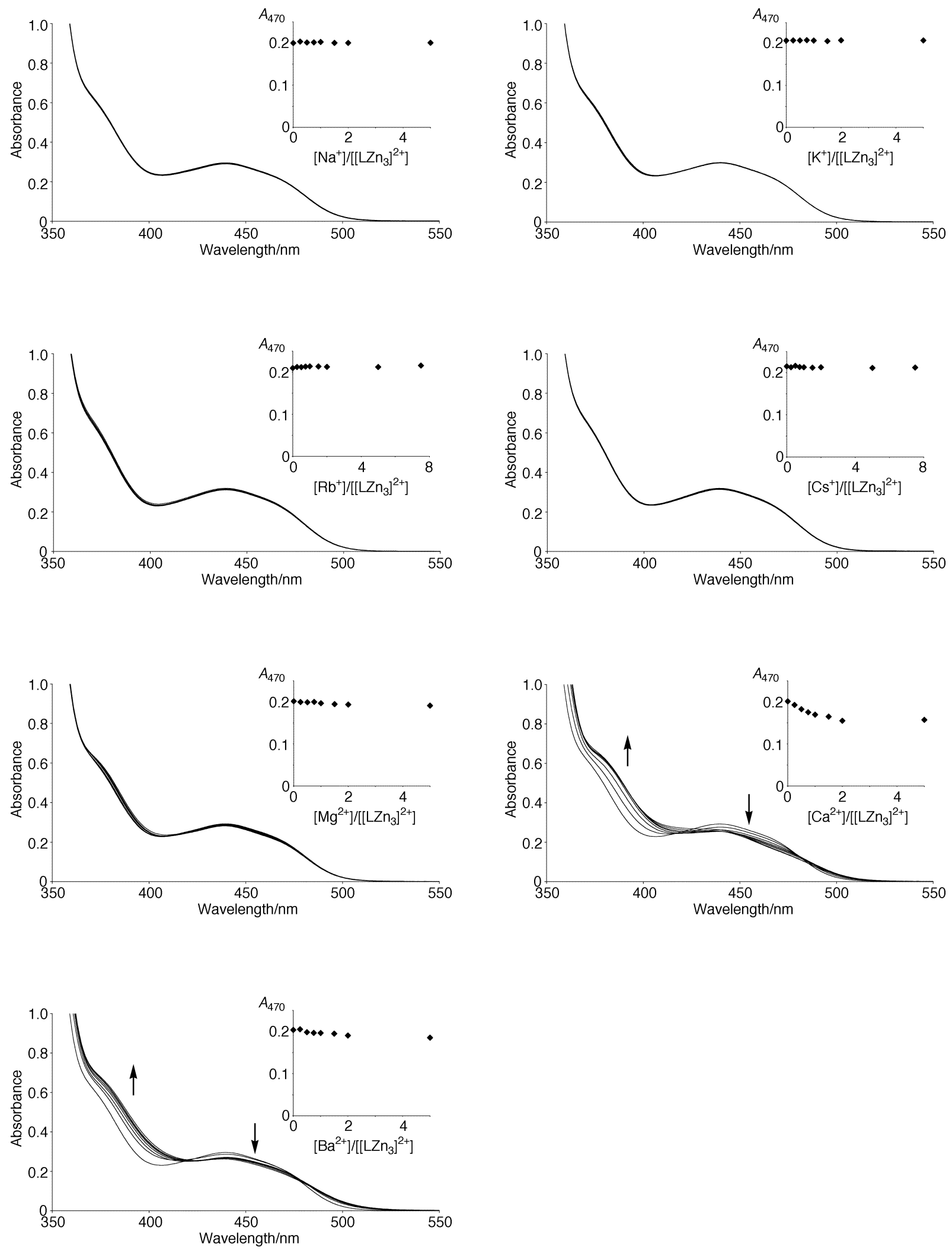

Figure S4. Spectrophotometric titration for the complexation between $\left[\mathrm{LZn}_{3}\right]^{2+}$ and $\mathrm{G}\left(\mathrm{ClO}_{4}\right)_{n}(\mathrm{G}$ $=\mathrm{Na}, \mathrm{K}, \mathrm{Rb}, \mathrm{Cs}, \mathrm{Mg}, \mathrm{Ca}, \mathrm{Ba})$ in chloroform/methanol $(1: 1)$. Concentration of $\left[\mathrm{LZn}_{3}\right]^{2+}$ is $0.1 \mathrm{mM}$. 


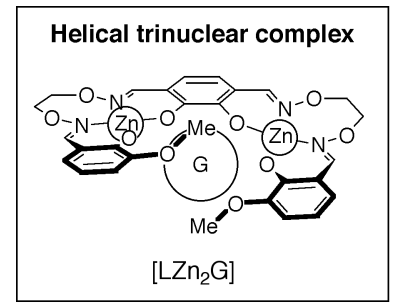

A (G: 10-coordinate)

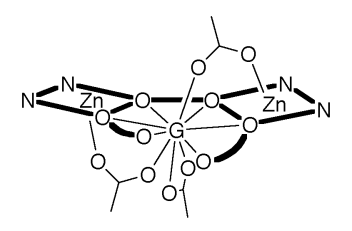

B (G: 9-coordinate)

B2

B1

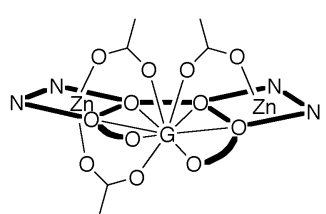

B3

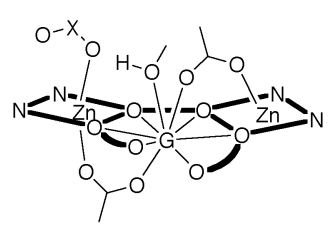

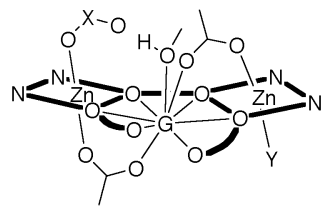

B1/B3 disordered

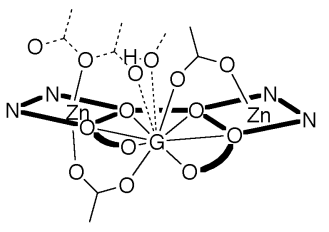

C (G: 8-coordinate)

C1 (closed type)

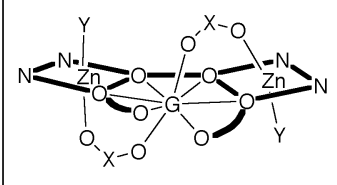

C2 (open type)

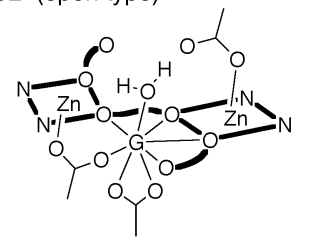

Scheme S1. Schematic representation of the crystal structures of helical trinuclear complexes $\left[\mathrm{LZn}_{2} \mathrm{G}\right]^{n+}$ showing the coordination mode of counter anions and solvent molecules. O-X-O and $\mathrm{Y}$ denote bridging anions $\left(\mathrm{OAc}^{-}, \mathrm{NO}_{3}^{-}, \mathrm{ClO}_{4}^{-}\right)$and coordinating solvent $\left(\mathrm{H}_{2} \mathrm{O}, \mathrm{MeOH}, \mathrm{EtOH}\right)$, respectively. 


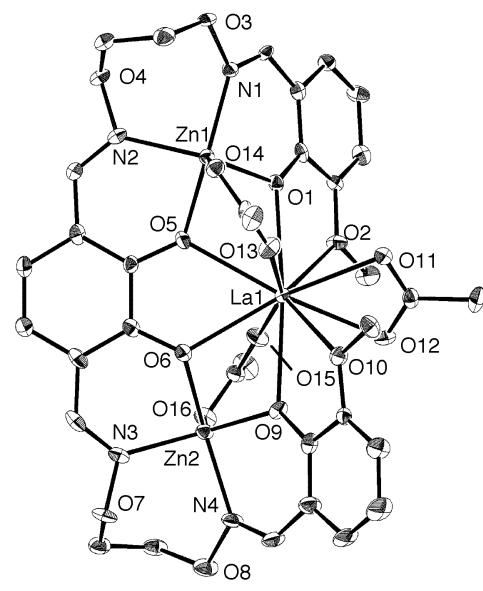

$\left[\mathrm{LZn}_{2} \mathrm{La}(\mathrm{OAc})_{3}\right]$

(Type A)

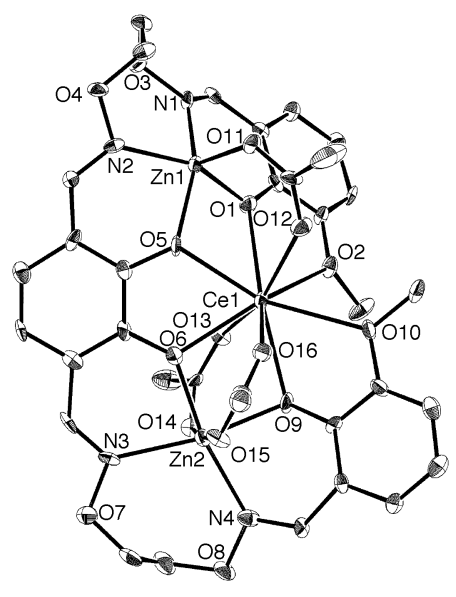

$\left[\mathrm{LZn}_{2} \mathrm{Ce}(\mathrm{OAc})_{3}\right]$ (Type B1/B3)

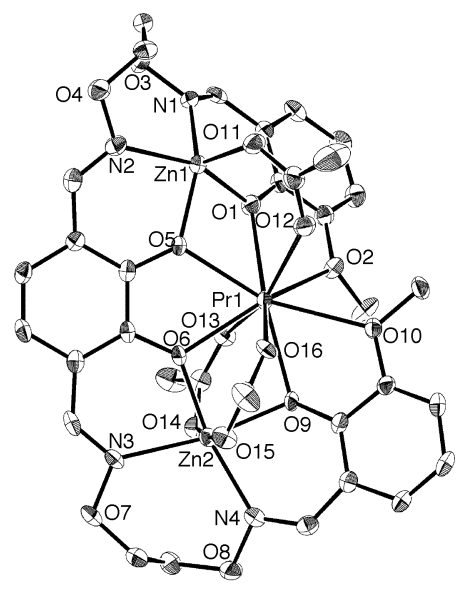

$\left[\mathrm{LZn} \mathrm{P}_{2} \mathrm{Pr}(\mathrm{OAC})_{3}\right]$
$(\mathrm{Type} \mathrm{B1/B3)}$

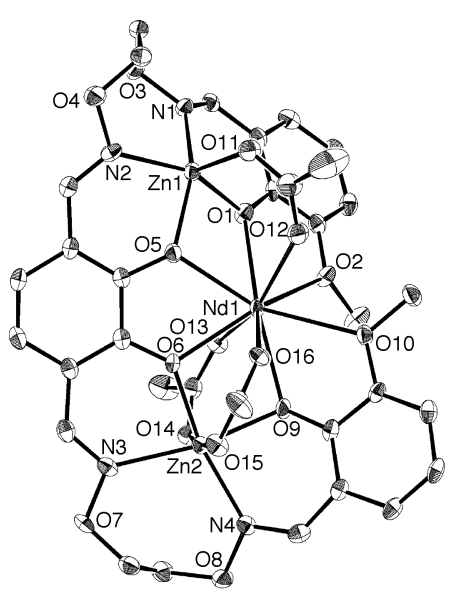

$\left[\mathrm{LZn} \mathrm{n}_{2} \mathrm{Nd}(\mathrm{OAc})_{3}\right]$
(Type B1/B3)

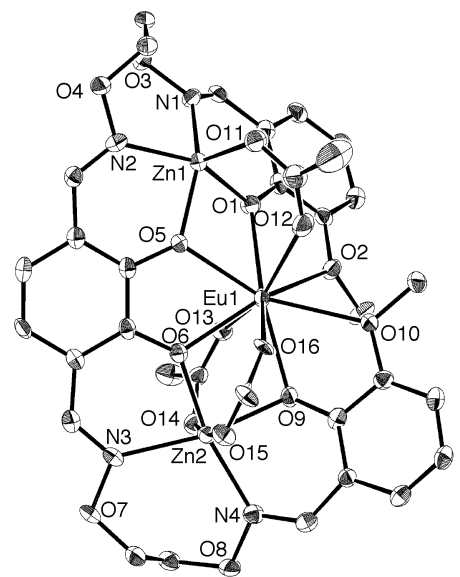

$\left[\mathrm{LZn}_{2} \mathrm{Eu}(\mathrm{OAc})_{3}\right]$

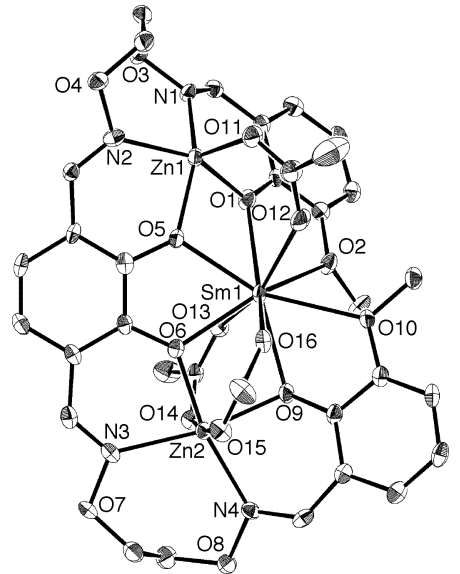

$\underset{\text { (Type B1/B3) }}{\left[\mathrm{LZn} \mathrm{S}_{2} \mathrm{Sm}(\mathrm{OAC})_{3}\right]}$

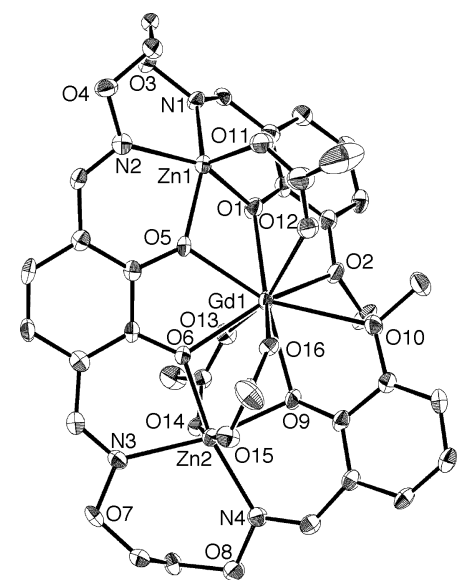

$\left[\mathrm{LZn} \mathrm{n}_{2} \mathrm{Gd}(\mathrm{OAC})_{3}\right]$

(Type B1/B3)

Figure S5. Crystal structure of heterotrinuclear complexes $\left[\mathrm{LZn}_{2} \mathrm{G}\right](\mathrm{G}=\mathrm{La}, \mathrm{Ce}, \mathrm{Pr}, \mathrm{Nd}, \mathrm{Sm}, \mathrm{Eu}$, $\mathrm{Gd).}$ 


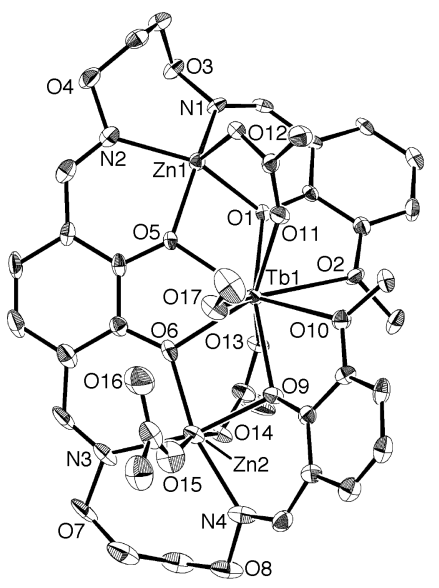

$\left[\mathrm{LZn}_{2} \mathrm{~Tb}(\mathrm{OAc})_{3}(\mathrm{MeOH})\right]$

(Type B2)

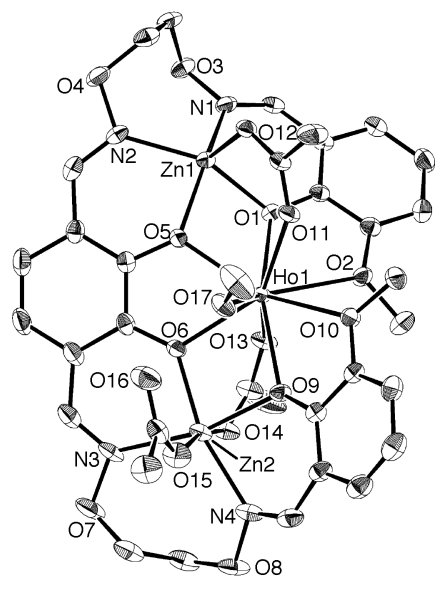

$\left[\mathrm{LZn}_{2} \mathrm{Ho}(\mathrm{OAc})_{3}(\mathrm{MeOH})\right]$
$($ Type B2)

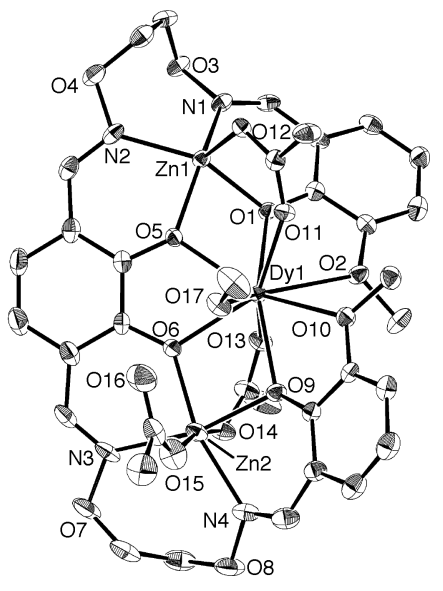

$\left[\mathrm{LZn}_{2} \mathrm{Dy}(\mathrm{OAc})_{3}(\mathrm{MeOH})\right]$

(Type B2)

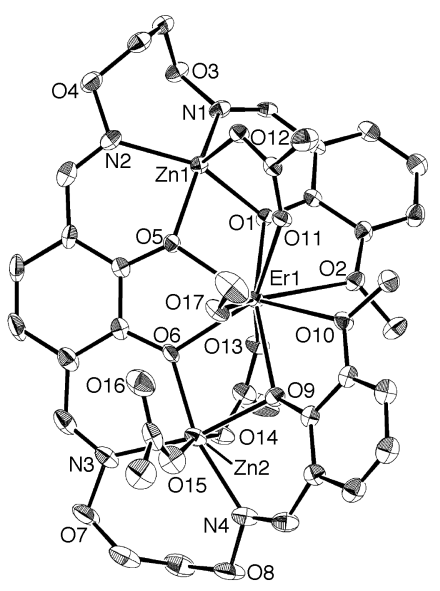

$\left[\mathrm{LZn}_{2} \mathrm{Er}(\mathrm{OAc})_{3}(\mathrm{MeOH})\right]$ (Type B2)

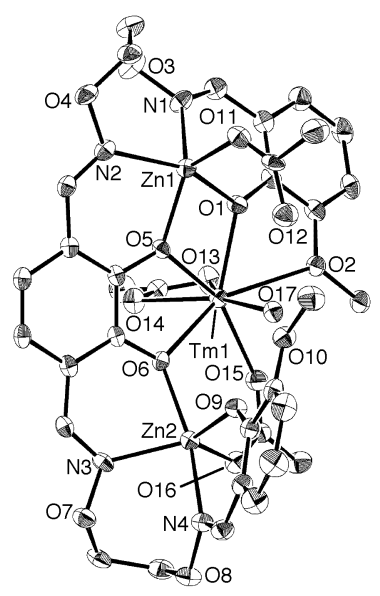

$\left[\mathrm{LZn}_{2} \mathrm{Tm}(\mathrm{OAc})_{3}\left(\mathrm{H}_{2} \mathrm{O}\right)\right]$

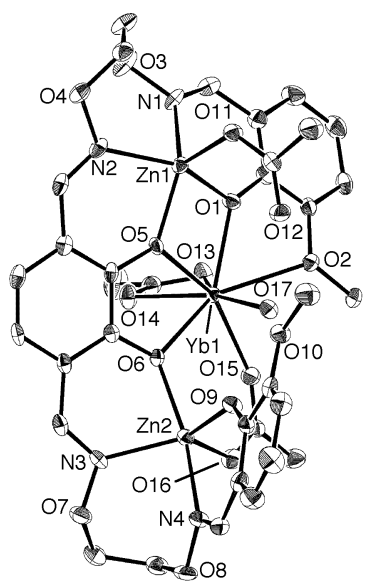

$\left[\mathrm{LZn}_{2} \mathrm{Yb}(\mathrm{OAc})_{3}\left(\mathrm{H}_{2} \mathrm{O}\right)\right]$

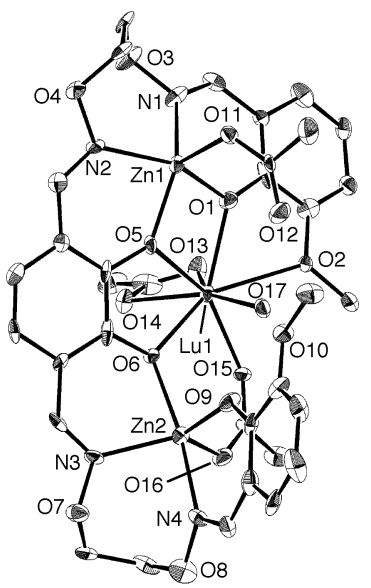

$\left[\mathrm{LZn}_{2} \mathrm{Lu}(\mathrm{OAc})_{3}\left(\mathrm{H}_{2} \mathrm{O}\right)\right]$

Figure S6. Crystal structure of heterotrinuclear complexes [ $\left.\mathrm{LZn}_{2} \mathrm{G}\right](\mathrm{G}=\mathrm{Tb}, \mathrm{Dy}, \mathrm{Ho}, \mathrm{Er}, \mathrm{Tm}, \mathrm{Yb}$, $\mathrm{Lu})$. 

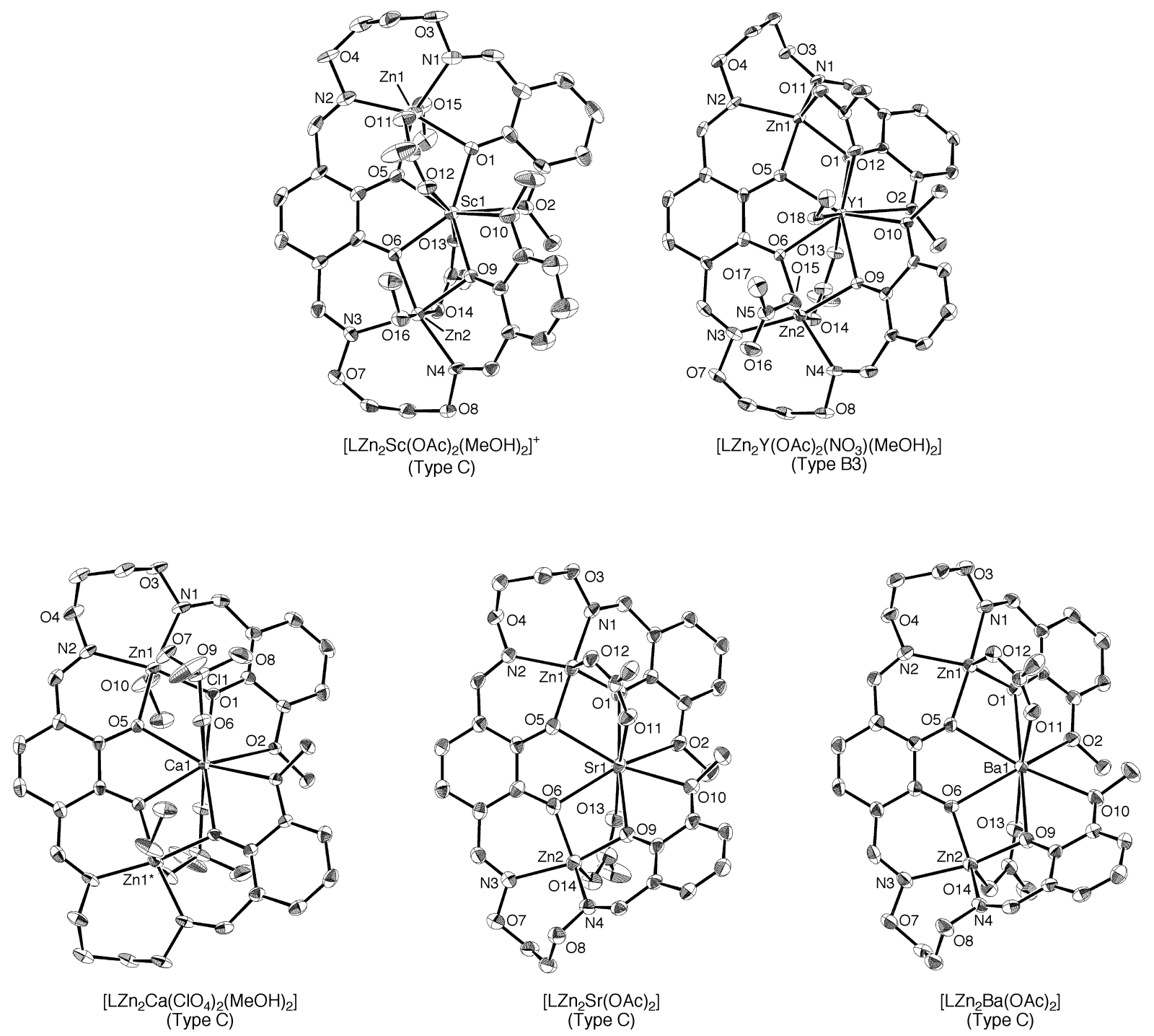

Figure S7. Crystal structure of heterotrinuclear complexes $\left[\mathrm{LZn}_{2} \mathrm{G}\right](\mathrm{G}=\mathrm{Ca}, \mathrm{Sr}, \mathrm{Ba})$. 
Table S1. Crystallographic data of trinuclear complexes

\begin{tabular}{lllll}
\hline Compound & $\begin{array}{l}\mathrm{LZn}_{3}(\mathrm{OAc})_{2} \\
\left(\mathrm{H}_{2} \mathrm{O}\right) \cdot \mathrm{Me}_{2} \mathrm{CO}\end{array}$ & $\begin{array}{l}{\left[\mathrm{L}_{2} \mathrm{Zn}_{3}(\mathrm{OAc})_{2}\right.} \\
\left(\mathrm{H}_{2} \mathrm{O}\right) \cdot 2 \mathrm{Me}_{2} \mathrm{CO}\end{array}$ & $\begin{array}{l}\mathrm{LZn}_{2} \mathrm{La}(\mathrm{OAc})_{3} \\
\bullet 2 \mathrm{CHCl}_{3}\end{array}$ & $\begin{array}{l}\mathrm{LZn}_{2} \mathrm{Ce}(\mathrm{OAc})_{3} \\
\bullet 3 \mathrm{MeOH} \bullet \mathrm{CHCl}_{3}\end{array}$ \\
\hline Crystal system & monoclinic & triclinic & monoclinic & monoclinic \\
Space group & $P 2_{1} / c$ & $P \overline{1}$ & $P 2_{1} / c$ & $C 2 / c$ \\
$a / \AA$ & $9.891(2)$ & $12.2709(6)$ & $10.585(4)$ & $35.296(19)$ \\
$b / \AA$ & $25.669(3)$ & $12.4998(8)$ & $12.885(4)$ & $13.172(6)$ \\
$c / \AA$ & $17.0297(10)$ & $14.6676(7)$ & $33.765(11)$ & $23.548(13)$ \\
$\alpha /$ deg & & $95.040(2)$ & & \\
$\beta /$ deg & $110.1817(7)$ & $104.2689(11)$ & $98.928(4)$ & $121.983(6)$ \\
$\gamma / \mathrm{deg}$ & & $112.4276(6)$ & & \\
$V / \AA^{3}$ & $4058.1(10)$ & $1973.47(19)$ & $4549(3)$ & $9286(8)$ \\
$Z$ & 4 & 2 & 4 & 8 \\
$R 1^{a}(I>2 \sigma(I))$ & 0.0644 & 0.0287 & 0.0720 & 0.0909 \\
$w R 2^{a}($ all data $)$ & 0.1325 & 0.0769 & 0.1703 & 0.2439 \\
\hline${ }^{a} R 1=\Sigma\left\|F_{\mathrm{o}} \mathrm{I}-\left|F_{\mathrm{c}} \| / \Sigma\right| F_{\mathrm{o}} \mathrm{I}(I>2 \sigma(I))\right.$. & ${ }^{b} w R 2=\left[\Sigma\left(w\left(F_{\mathrm{o}}{ }^{2}-F_{\mathrm{c}}{ }^{2}\right)^{2}\right) / \Sigma\left(w\left(F_{\mathrm{o}}{ }^{2}\right)^{2}\right)\right]^{1 / 2}($ all data $)$.
\end{tabular}

Table S1 (continued).

\begin{tabular}{|c|c|c|c|c|}
\hline Compound & $\begin{array}{l}\mathrm{LZn}_{2} \mathrm{Pr}(\mathrm{OAc})_{3} \bullet \\
3 \mathrm{MeOH} \bullet \mathrm{CHCl}_{3} \bullet \\
0.25 \mathrm{H}_{2} \mathrm{O}\end{array}$ & $\begin{array}{l}\mathrm{LZn}_{2} \mathrm{Nd}(\mathrm{OAc})_{3} \bullet \\
3 \mathrm{MeOH} \bullet \mathrm{CHCl}_{3} \bullet \\
0.25 \mathrm{H}_{2} \mathrm{O}\end{array}$ & $\begin{array}{l}\mathrm{LZn}_{2} \mathrm{Sm}(\mathrm{OAc})_{3} \bullet \\
3 \mathrm{MeOH} \bullet \mathrm{CHCl}_{3}\end{array}$ & $\begin{array}{l}\mathrm{LZn}_{2} \mathrm{Eu}(\mathrm{OAc})_{3} \bullet \\
3 \mathrm{MeOH} \cdot \mathrm{CHCl}_{3}\end{array}$ \\
\hline Crystal system & monoclinic & monoclinic & monoclinic & monoclinic \\
\hline Space group & $C 2 / c$ & $C 2 / c$ & $C 2 / c$ & $C 2 / c$ \\
\hline$a / \AA$ & $35.42(2)$ & $35.185(12)$ & $35.331(16)$ & $35.388(14)$ \\
\hline$b / \AA$ & $13.147(8)$ & $13.183(4)$ & $13.172(6)$ & $13.185(5)$ \\
\hline$c / \AA$ & $23.595(15)$ & $23.446(8)$ & $23.515(11)$ & $23.550(9)$ \\
\hline \multicolumn{5}{|l|}{$\alpha / \operatorname{deg}$} \\
\hline$\beta / \operatorname{deg}$ & $122.2609(17)$ & $121.9283(13)$ & $121.782(5)$ & $121.860(4)$ \\
\hline \multicolumn{5}{|l|}{$\gamma / \operatorname{deg}$} \\
\hline$V / \AA^{3}$ & $9292(10)$ & $9230(5)$ & $9303(7)$ & $9333(6)$ \\
\hline$Z$ & 8 & 8 & 8 & 8 \\
\hline$R 1^{a}(I>2 \sigma(I))$ & 0.0488 & 0.0400 & 0.0567 & 0.0510 \\
\hline$w R 2^{a}$ (all data) & 0.1186 & 0.1089 & 0.1518 & 0.1271 \\
\hline
\end{tabular}


Table S1. continued

\begin{tabular}{|c|c|c|c|c|}
\hline Compound & $\begin{array}{l}\mathrm{LZn}_{2} \mathrm{Gd}(\mathrm{OAc})_{3} \bullet \\
2.8 \mathrm{MeOH} \bullet \mathrm{CHCl}_{3} \bullet \\
0.5 \mathrm{H}_{2} \mathrm{O}\end{array}$ & $\begin{array}{l}\mathrm{LZn}_{2} \mathrm{~Tb}(\mathrm{OAc})_{3} \bullet \\
\mathrm{MeOH} \bullet \mathrm{H}_{2} \mathrm{O} \bullet \mathrm{Et}_{2} \mathrm{O}\end{array}$ & $\begin{array}{l}\mathrm{LZn}_{2} \mathrm{Dy}(\mathrm{OAc})_{3} \bullet \\
\mathrm{MeOH} \bullet \mathrm{H}_{2} \mathrm{O} \bullet \mathrm{Et}_{2} \mathrm{O}\end{array}$ & $\begin{array}{l}\mathrm{LZn}_{2} \mathrm{Ho}(\mathrm{OAc})_{3} \bullet \\
\mathrm{MeOH} \bullet \mathrm{H}_{2} \mathrm{O} \bullet \mathrm{Et}_{2} \mathrm{O}\end{array}$ \\
\hline Crystal system & monoclinic & triclinic & triclinic & triclinic \\
\hline Space group & $C 2 / c$ & $P \overline{1}$ & $P \overline{1}$ & $P \overline{1}$ \\
\hline$a / \AA ̊$ & $35.270(10)$ & $12.948(9)$ & $13.019(7)$ & $13.0652(9)$ \\
\hline$b / \AA$ & $13.185(3)$ & $13.222(8)$ & $13.279(6)$ & $13.2752(8)$ \\
\hline$c / \AA$ & $23.448(7)$ & $13.435(8)$ & $13.444(7)$ & $13.4401(7)$ \\
\hline$\alpha / \operatorname{deg}$ & & $73.867(19)$ & $74.158(12)$ & $74.310(3)$ \\
\hline$\beta /$ deg & $121.6798(11)$ & $83.20(2)$ & $83.194(14)$ & $83.016(2)$ \\
\hline$\gamma / \operatorname{deg}$ & & $86.92(2)$ & $87.200(16)$ & $87.0386(19)$ \\
\hline$V / \AA^{3}$ & $9279(4)$ & 2193(2) & $2219.8(19)$ & $2227.1(2)$ \\
\hline$Z$ & 8 & 2 & 2 & 2 \\
\hline$R 1^{a}(I>2 \sigma(I))$ & 0.0467 & 0.0546 & 0.0439 & 0.0448 \\
\hline$w R 2^{a}$ (all data) & 0.0974 & 0.1409 & 0.1131 & 0.1213 \\
\hline
\end{tabular}

Table S1. continued

\begin{tabular}{lllll}
\hline Compound & $\begin{array}{l}\mathrm{LZn}_{2} \mathrm{Er}(\mathrm{OAc})_{3} \bullet \\
\mathrm{MeOH} \bullet \mathrm{H}_{2} \mathrm{O} \bullet \mathrm{Et}_{2} \mathrm{O}\end{array}$ & $\begin{array}{l}\mathrm{LZn}_{2} \mathrm{Tm}(\mathrm{OAc})_{3} \bullet \\
\mathrm{H}_{2} \mathrm{O} \bullet \mathrm{CHCl}_{3}\end{array}$ & $\begin{array}{l}\mathrm{LZn}_{2} \mathrm{Yb}(\mathrm{OAc})_{3} \bullet \\
\mathrm{H}_{2} \mathrm{O} \bullet \mathrm{CHCl}_{3}\end{array}$ & $\begin{array}{l}\mathrm{LZn}_{2} \mathrm{Lu}(\mathrm{OAc})_{3} \bullet \\
\mathrm{H}_{2} \mathrm{O} \bullet \mathrm{Me}_{2} \mathrm{CO}\end{array}$ \\
\hline Crystal system & triclinic & monoclinic & monoclinic & monoclinic \\
Space group & $P \overline{1}$ & $P 2_{1} / n$ & $P 2_{1} / n$ & $P 2_{1} / n$ \\
$a / \AA$ & $13.027(6)$ & $13.6707(12)$ & $13.646(3)$ & $13.675(8)$ \\
$b / \AA$ & $13.282(6)$ & $15.3874(10)$ & $15.274(3)$ & $15.847(8)$ \\
$c / \AA$ & $13.479(6)$ & $19.9724(14)$ & $19.810(4)$ & $18.766(10)$ \\
$\alpha /$ deg & $74.017(12)$ & & & \\
$\beta /$ deg & $83.078(14)$ & $94.805(2)$ & $95.0519(8)$ & $95.454(8)$ \\
$\gamma /$ deg & $86.972(14)$ & & & \\
$V / \AA^{3}$ & $2225.2(17)$ & $4186.6(5)$ & $4112.8(15)$ & $4048(4)$ \\
$Z$ & 2 & 4 & 4 & 4 \\
$R 1^{a}(I>2 \sigma(I))$ & 0.0422 & 0.0563 & 0.0406 & 0.0893 \\
$w R 2^{a}($ all data $)$ & 0.1104 & 0.1454 & 0.1114 & 0.2168 \\
\hline${ }^{a} R 1=\Sigma\left\|F_{\mathrm{o}} \mathrm{I}-\left|F_{\mathrm{c}} \| / \Sigma\right| F_{\mathrm{o}} \mathrm{I}(I>2 \sigma(I))\right.$. & ${ }^{b} w R 2=\left[\Sigma\left(w\left(F_{\mathrm{o}}{ }^{2}-F_{\mathrm{c}}{ }^{2}\right)^{2}\right) / \Sigma\left(w\left(F_{\mathrm{o}}{ }^{2}\right)^{2}\right)\right]^{1 / 2}($ all data $)$.
\end{tabular}


Table S1. continued

\begin{tabular}{|c|c|c|c|c|c|}
\hline Compound & $\begin{array}{l}\mathrm{LZn}_{2} \mathrm{Sc}(\mathrm{OAc})_{2} \\
\left(\mathrm{NO}_{3}\right) \cdot 2 \mathrm{MeOH} \bullet \\
\mathrm{EtOH}\end{array}$ & $\begin{array}{l}\mathrm{LZn}_{2} \mathrm{Y}(\mathrm{OAc})_{2} \\
\left(\mathrm{NO}_{3}\right) \cdot 2 \mathrm{MeOH}\end{array}$ & $\begin{array}{l}\mathrm{LZn}_{2} \mathrm{Ca}- \\
\left(\mathrm{ClO}_{4}\right)_{2} \\
2 \mathrm{MeOH}\end{array}$ & $\begin{array}{l}\mathrm{LZn}_{2} \mathrm{Sr}(\mathrm{OAc})_{2} \bullet \\
\mathrm{CH}_{2} \mathrm{Cl}_{2}\end{array}$ & $\begin{array}{l}\mathrm{LZn}_{2} \mathrm{Ba}(\mathrm{OAc})_{2} \bullet \\
0.5 \mathrm{CHCl}_{3} \bullet \\
\mathrm{Et}_{2} \mathrm{O} \bullet 1.5 \mathrm{H}_{2} \mathrm{O}\end{array}$ \\
\hline Crystal system & triclinic & triclinic & monoclinic & triclinic & triclinic \\
\hline Space group & $P \overline{1}$ & $P \overline{1}$ & $C 2 / c$ & $P \overline{1}$ & $P \overline{1}$ \\
\hline$a / \AA ̊$ & $12.297(6)$ & $12.380(5)$ & $10.824(4)$ & $11.991(2)$ & $13.1572(5)$ \\
\hline$b / \AA ̊$ & $16.082(8)$ & $12.893(6)$ & $15.726(6)$ & $12.906(3)$ & $13.8486(12)$ \\
\hline$c / \AA ̊$ & $21.117(10)$ & $13.200(7)$ & $22.462(9)$ & $14.366(4)$ & $15.7800(9)$ \\
\hline$\alpha / \operatorname{deg}$ & $95.050(8)$ & $88.920(14)$ & & $67.548(5)$ & $110.130(3)$ \\
\hline$\beta / \operatorname{deg}$ & $95.675(9)$ & $85.990(16)$ & $92.693(6)$ & 72.491(7) & $105.072(3)$ \\
\hline$\gamma / \mathrm{deg}$ & $94.160(7)$ & $67.455(10)$ & & $84.174(9)$ & $106.476(3)$ \\
\hline$V / \AA^{3}$ & $4125(4)$ & $1941.0(16)$ & $3819(3)$ & $1959.2(7)$ & $2375.8(3)$ \\
\hline$Z$ & 4 & 2 & 4 & 2 & 2 \\
\hline$R 1^{a}(I>2 \sigma(I))$ & 0.0782 & 0.0504 & 0.0378 & 0.0667 & 0.0747 \\
\hline$w R 2^{a}$ (all data) & 0.1995 & 0.1274 & 0.0899 & 0.1690 & 0.2014 \\
\hline
\end{tabular}

